\title{
Prognostic significance of membrane-associated mucins 1 and 4 in gastric adenocarcinoma
}

\author{
ILSEON HWANG ${ }^{1,3}$, YU NA KANG ${ }^{1}$, JIN YOUNG KIM $^{2,3}$, YOUNG ROK DO ${ }^{2}$, \\ HONG SUK SONG ${ }^{2,3}$ and KEON UK PARK ${ }^{2,3}$
}

\author{
${ }^{1}$ Department of Pathology and ${ }^{2}$ Division of Hematology/Oncology, Department of Internal Medicine; \\ ${ }^{3}$ Pain Research Center, School of Medicine, Keimyung University, Jung-gu, Daegu 700-712, Republic of Korea
}

Received March 9, 2012; Accepted May 28, 2012

DOI: $10.3892 /$ etm.2012.598

\begin{abstract}
Aberrant expression of mucins is likely associated with cancer biology as alterations in the expression and/or glycosylation patterns of various mucins have been noted. Expression of the mucin family in gastric cancers has been reported in numerous studies, but the results are conflicting. Therefore, we investigated the potential use of mucin (MUC)1 and 4 as prognostic markers in gastric cancer according to histological subtype. Three-hundred and sixty-five gastric adenocarcinoma patients who underwent surgical resection were selected for this study. Among the 365 gastric cancer samples tested here, $34 \%$ consisted of early gastric cancer and $66 \%$ were advanced. In terms of location, $68.7 \%$ of the cohort had intestinal-type cancer and $30.7 \%$ had diffusetype. We constructed tissue microarrays with formalin-fixed paraffin-embedded blocks of gastric cancer and these microarrays were evaluated for phenotypic expression of MUC1/4 using monoclonal antibodies. Two-hundred and ninety-two patients $(92.7 \%)$ were positive for MUC1 and $216(60.5 \%)$ were positive for MUC4. MUC1 expression was not correlated with any other clinicopathological variables such as age, gender, depth of invasion, lymph node metastasis, Lauren classification or recurrence. However, loss of MUC4 expression was significantly correlated with recurrence $(\mathrm{p}=0.033)$. MUC4 expression was also significantly correlated with better disease-free survival $(p=0.049)$ and particularly in the intestinal-type $(\mathrm{p}=0.018)$. Our present findings demonstrated that loss of MUC4 expression can be used as a prognostic marker in gastric cancer. Loss of MUC4 expression is a prognostic indicator of increased recurrence and poor disease-free survival in patients with gastric cancer.
\end{abstract}

Correspondence to: Professor Keon Uk Park, Division of Hematology/Oncology, Department of Internal Medicine, School of Medicine, Keimyung University, Dalseong-ro 56, Jung-gu, Daegu 700-712, Republic of Korea

E-mail: keonukpark@gmail.com

Key words: gastric cancer, mucin 4, prognosis

\section{Introduction}

Mucins are high-molecular-weight epithelial glycoproteins that are heavily glycosylated with numerous oligosaccharide side chains linked to a protein backbone called apomucin. Mucin proteins are known for providing protection and lubrication to epithelial surfaces; in addition, their roles in cell signaling are under intense study (1-2). Aberrant expression of mucins is likely associated with cancer biology as alterations in the expression and/or glycosylation patterns of various mucins influence cellular growth, differentiation, transformation, adhesion, invasion and immune surveillance. Mucins are divided into two distinct classes according to their structure and function (3). Secreted-type mucins (MUC2, 5AC, 5B, 6, 7, 8,9 and 19) are glycoproteins constituting the major macromolecular component of mucus, and membrane-associated type mucins (MUC1, 3A, 3B, 4, 12, 13, 15, 16, 17 and 20) contribute to epithelial cell-cell interactions (3-4).

MUC1 is normally abundantly present at the luminal surface of various secretory epithelial cells (5), and is associated with the epidermal growth factor receptor tyrosine kinase and is involved in cellular signaling $(1,6)$. Overexpression of MUC1 is reported in malignant neoplasms including breast, pancreatic, colorectal or non-small-cell carcinoma of the lung, and is associated with a poor prognosis (7-9).

MUC4 expression is also present in normal epithelial tissues including stomach, colon, cervix and lung. The overexpression of MUC4 has been discovered in a number of human neoplasms (10-12). It has been shown that MUC4 expression is related to aggressive tumor behavior or a poor outcome in intrahepatic cholangiocarcinoma, invasive ductal carcinoma of the pancreas, extrahepatic bile duct carcinoma, and lung adenocarcinoma (13-17). On the other hand, loss of MUC4 expression is related to poor survival in mucoepidermoid carcinoma of the salivary gland, squamous cell carcinoma of the upper aerodigestive system, and adenocarcinoma of lung (18-21). The function of MUC4 in gastric cancer is not understood. To date, there have been few studies on the relationship between MUC4 expression and patient prognosis in gastric adenocarcinoma.

The aims of this study were to investigate MUC1 and 4 expression patterns and to evaluate the correlation of MUC1 and 4 expression with patient prognosis in gastric adenocarcinoma. 


\section{Materials and methods}

Patients. We recruited 365 patients who underwent gastrectomy for gastric adenocarcinoma from archives of paraffin blocks at Keimyung University Dongsan Hospital from October 1995 to December 1999. Tissue samples were fixed in formalin and embedded in paraffin. All cases were reviewed by an expert panel of two pathologists according to the current criteria of the WHO classification for morphological features and immunohistochemical results. The clinical data (age, gender, $\mathrm{T}$ stage, $\mathrm{N}$ stage, Lauren classification, date of diagnosis, adjuvant chemotherapy, date of relapse, and date of last follow-up) and pathological reports of the patients with gastric adenocarcinoma were collected from the medical records. Patients were divided into subgroups: $\leq 60$ and $>60$ years of age.

Immunohistochemistry. Formalin-fixed, paraffin-embedded tissue samples were used for tissue microarray (TMA). Representative areas of each tumor were marked on each $\mathrm{H} \& \mathrm{E}$-stained slide, and the corresponding areas of tissue blocks were sampled. The designated areas of each donor block were collected using a tissue cylinder punch of 5-mm diameter, and samples were transferred to a recipient block.

Sections $(4 \mu \mathrm{m})$ from TMAs were cut from $10 \%(\mathrm{v} / \mathrm{v})$ formalin buffer and embedded in paraffin, were mounted on Superfrost Plus glass slides (VWR Scientific, West Chester, PA, USA) and incubated at $60^{\circ} \mathrm{C}$ for $15 \mathrm{~min}$. The slides were next deparaffinized in xylene, rehydrated in graded alcohol solutions, and washed in tap water. Endogenous peroxidase activity was blocked by the addition of $3 \%(\mathrm{v} / \mathrm{v}) \mathrm{H}_{2} \mathrm{O}_{2}$. Slides were placed in a steam cooker filled with $10 \mathrm{mM}$ sodium citrate buffer, $\mathrm{pH}$ 6.0, for antigen retrieval. After treatment with a blocking agent (Dako, Carpinteria, CA, USA) for $10 \mathrm{~min}$ to block nonspecific protein binding, samples were incubated for a further $1 \mathrm{~h}$ with primary anti-human mouse antibodies which included MUC1 (1:200, Zymed, South San Francisco, CA, USA) and MUC4 (1:300, Zymed). After reaction with a biotinylated antibody for $30 \mathrm{~min}$, antigen-antibody complexes were visualized using a streptavidin-horseradish peroxidase conjugate (Dako LSAB kit; Dako, Los Angeles, CA, USA) employing diaminobenzidine as the chromogen. Slides were counterstained with Meyer's hematoxylin for 3-5 min.

Immunopositivity for MUC1 and MUC4 was evaluated by staining intensity ( 0 , negative; 1 , weak; 2 , moderate; 3 , strong) and the proportions of positive staining cells $(0,0 \%$ positive; $1, \leq 10 \%$ positive; $2,>10 \%$ and $\leq 50 \%$ positive; $3,>50 \%$ positive). Samples were considered positive for MUC1 or 4 when staining intensity was $>0$ (weak-to-strong) and $>10 \%$ of cells were positively stained.

Statistics. The SPSS statistical package, version 19.0 for Windows, was used for all statistical analyses. The relationship between MUC1/4 immunoreactivity and each clinicopathological variable was evaluated using the Chi-square or Fisher's exact test, as appropriate. Disease-free survival was measured from the date of diagnosis to the date of recurrence or the last follow-up . Overall survival was measured from the date of diagnosis to the date of death or the last follow-up visit. Diseasefree and overall survival were measured according to the
Table I. Clinicopathological variables of the gastric adenocarcinoma cases.

\begin{tabular}{lc}
\hline Clinical variables & Value \\
\hline Mean age, years (range) & $56.2(25-82)$ \\
Gender (M/F) & $248: 117$ \\
Depth of tumor invasion, $\mathrm{n}(\%)$ & \\
$\quad \begin{array}{l}\text { Mucosa or submucosa } \\
\text { (early gastric cancer) }\end{array}$ & $126(34.5)$ \\
Other than submucosa & \\
$\quad$ (advanced gastric cancer) & $239(65.5)$ \\
Lymph node (regional) metastasis, $\mathrm{n}(\%)$ & \\
$\quad$ No & $182(49.9)$ \\
$\quad$ Yes & $182(50.1)$ \\
Lauren classification, $\mathrm{n}(\%)$ & \\
$\quad$ Diffuse-type & $112(30.7)$ \\
Intestinal-type & $251(68.8)$ \\
$\quad$ Mixed-type & $2(0.5)$ \\
Recurrence, $\mathrm{n}(\%)$ & $89(24.4)$ \\
Survival, $\mathrm{n}(\%)$ & $72(19.7)$ \\
\hline
\end{tabular}

Kaplan-Meier method and the Cox regression test, and survival rates were compared using the log-rank test. P-values $<0.05$ were considered to indicate statistically significant results, and all p-values correspond to two-sided significance tests.

\section{Results}

Clinicopathological variables. The mean age of the 365 patients with gastric adenocarcinoma was 56.2 years (range, 25-82 years). There were 248 (67.9\%) male patients and $117(32.1 \%)$ female patients. Early gastric carcinoma, invading the mucosal or submucosal layer, was observed in $126(34.5 \%)$ patients and advanced gastric carcinoma, which invaded the proper muscle or a deeper layer, was observed in 239 (65.5\%). According to the Lauren classification, 112 (30.7\%) patients had diffuse type, $251(68.8 \%)$ had intestinal type and two $(0.5 \%)$ had mixed type. The clinicopathological variables of gastric adenocarcinoma are summarized in Table I.

MUC1 and MUC4 expression. MUC1 demonstrated diffuse and strong immunopositivity on the membrane and in the cytoplasm of normal gastric glandular cells. MUC4 was also strongly positive on the membrane and in the cytoplasm of normal surface glandular cells, but was negative on the membrane or in the cytoplasm of pyloric glandular cells.

Two hundred and ninety-two out of 315 (92.7\%) cases were positive for MUC1 immunoreactivity and 216 out of 317 (60.5\%) were positive for MUC4 immunoreactivity. Staining patterns of MUC1 and MUC4 in tumor cells were the same as that of MUC1 and MUC4 in normal glandular cells (Fig. 1 and 2). MUC1 expression was not correlated with any other clinicopathological variables such as age, gender, depth of invasion, lymph node metastasis, Lauren classification or recurrence. However, MUC4 expression was significantly 
A

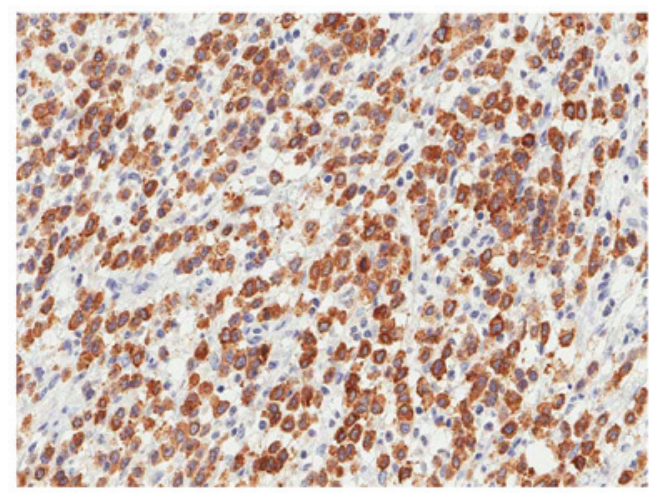

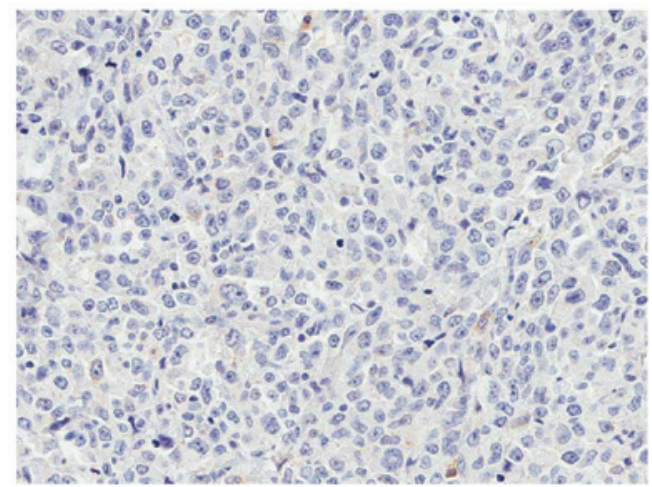

Figure 1. The majority of the gastric adenocarcinoma cases (92.7\%) showed MUC1 expression (A) while others exhibited no MUC1 expression (B).

A

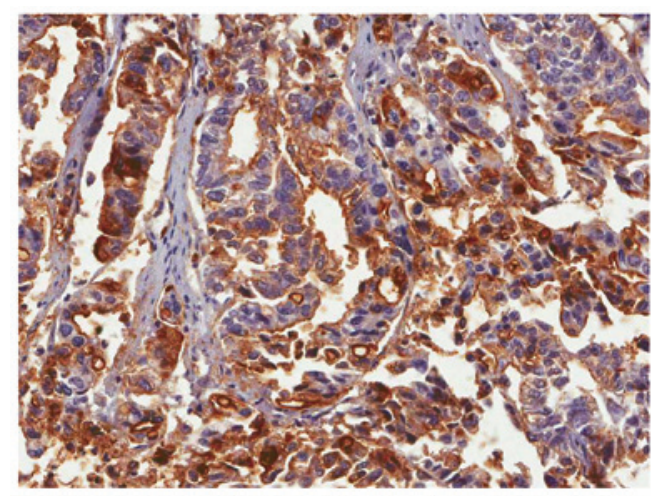

C

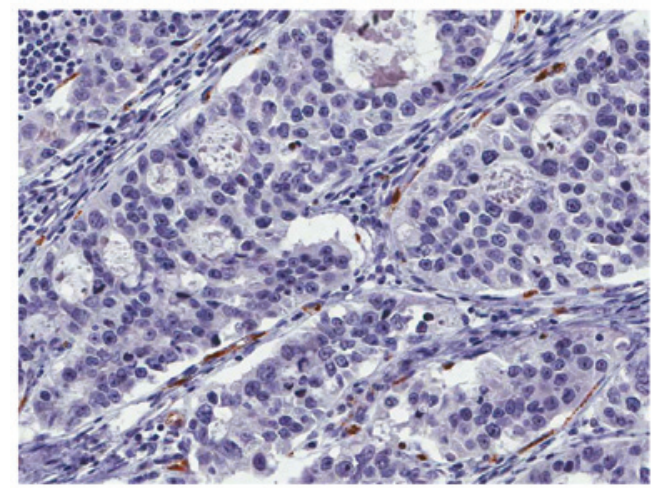

B

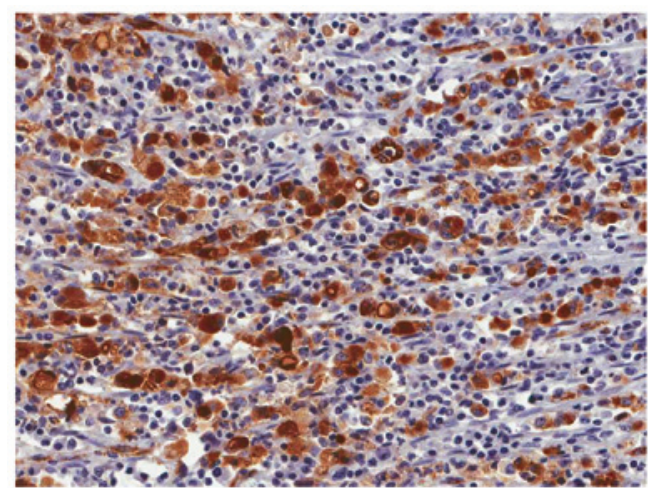

D

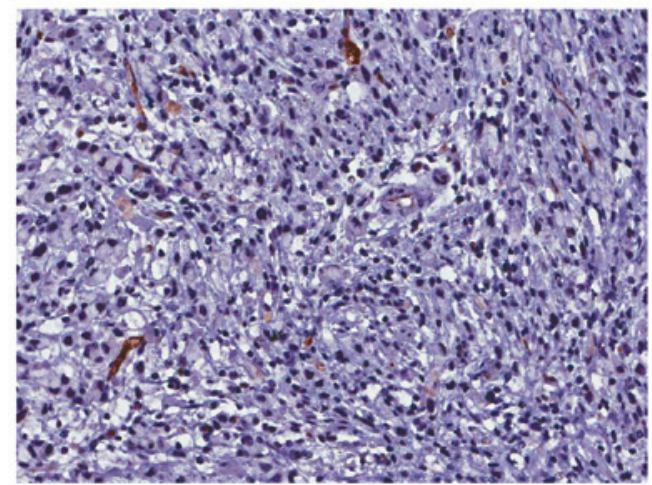

Figure 2. Some tumors of intestinal type were positive for MUC4 (A) while other tumors of intestinal type were negative for MUC4 (C). There was no morphological difference in MUC4-positive and MUC4-negative tumor cells of the intestinal type. Likewise in the diffuse type, some of the tumors were positive (B) while others were negative (D). Morphological differences in MUC4-positive and -negative tumor cells were not noted.

correlated with recurrence $(\mathrm{P}=0.033)$, but not with any other variables such as age, gender, depth of invasion, lymph node metastasis or Lauren classification (Table II). The prevalence of MUC4 expression was higher in the intestinal-type (42.4\%) than in the diffuse-type (32.7\%) but this did not achieve statistical significance $(\mathrm{P}=0.100)$. MUC4-positive tumor cells were not morphologically different than the MUC4-negative tumor cells in either the diffuse- or intestinal-type. (Fig. 2)

Prognosis. Loss of MUC4 expression was significantly correlated with worse disease-free survival $(\mathrm{p}=0.049)$ but was not significantly correlated with overall survival $(\mathrm{p}=0.671)$ in Kaplan-Meier survival test. (Fig. 3) Tumor invasion (more than submucosa), presence of lymph node metastasis, and diffuse type by Lauren classification were significantly correlated with worse disease-free survival $(\mathrm{p}<0.001,<0.001$ and $=0.044)$ in
Kaplan-Meier survival analysis. However, MUC1 expression and other clinical variables including age, gender, or adjuvant chemotherapy were not correlated with disease-free survival.

In intestinal-type gastric cancer according to Lauren classification, loss of MUC4 expression was significantly correlated with worse disease-free survival $(p=0.018)$, but was not significantly correlated with disease-free survival in the diffuse-type although loss of MUC4 was significantly correlated with disease-free survival in overall types of gastric carcinoma. Loss of MUC4 expression was also significantly correlated with worse disease-free survival $(\mathrm{p}=0.025)$ in the older age group $(>60)$, but was not correlated with worse disease-free survival in the younger age group $(\leq 60)$. Gender, depth of tumor invasion, lymph node metastasis, or adjuvant chemotherapy did not have an effect on MUC4 expression and disease-free survival. 
Table II. Correlations between MUC1/4 expression and clinical variables of the gastric adenocarcinoma cases.

\begin{tabular}{|c|c|c|c|c|c|}
\hline \multicolumn{2}{|c|}{ MUC1 expression ${ }^{a}$} & \multirow[b]{2}{*}{ P-value } & \multicolumn{2}{|c|}{ MUC4 expression ${ }^{\mathrm{a}}$} & \multirow[b]{2}{*}{ P-value } \\
\hline $\begin{array}{c}\text { Positive } \\
\text { No. }\end{array}$ & $\begin{array}{c}\text { Negative } \\
\text { No. }\end{array}$ & & $\begin{array}{c}\text { Positive } \\
\text { No. }\end{array}$ & $\begin{array}{c}\text { Negative } \\
\text { No. }\end{array}$ & \\
\hline
\end{tabular}

\begin{tabular}{|c|c|c|c|c|c|c|}
\hline \multicolumn{7}{|l|}{ Age (years) } \\
\hline$\leq 60$ & $14 / 315$ & $165 / 315$ & \multirow[t]{2}{*}{0.828} & $80 / 357$ & $130 / 357$ & \multirow[t]{2}{*}{0.582} \\
\hline$>60$ & $9 / 315$ & $127 / 315$ & & $61 / 357$ & $86 / 357$ & \\
\hline \multicolumn{7}{|l|}{ Gender } \\
\hline Male & $14 / 315$ & $199 / 315$ & \multirow[t]{2}{*}{0.492} & $98 / 357$ & $144 / 357$ & \multirow[t]{2}{*}{0.643} \\
\hline Female & $9 / 315$ & $93 / 315$ & & $43 / 357$ & $72 / 357$ & \\
\hline \multicolumn{7}{|l|}{ Depth of invasion } \\
\hline Early gastric cancer & $4 / 315$ & $93 / 315$ & \multirow[t]{2}{*}{0.168} & $40 / 357$ & $80 / 357$ & \multirow[t]{2}{*}{0.109} \\
\hline Advanced gastric cancer & $19 / 315$ & $199 / 315$ & & $101 / 357$ & $136 / 357$ & \\
\hline \multicolumn{7}{|l|}{ Lymph node metastasis } \\
\hline No & $10 / 315$ & $138 / 315$ & \multirow[t]{2}{*}{0.830} & $62 / 357$ & $113 / 357$ & \multirow[t]{2}{*}{0.131} \\
\hline Yes & $13 / 315$ & $154 / 315$ & & $79 / 357$ & $103 / 357$ & \\
\hline \multicolumn{7}{|l|}{ Lauren classification } \\
\hline Diffuse-type & $6 / 314$ & $93 / 314$ & \multirow[t]{2}{*}{0.647} & $36 / 355$ & $74 / 355$ & \multirow[t]{2}{*}{0.100} \\
\hline Intestinal-type & $17 / 314$ & $198 / 314$ & & $104 / 355$ & $141 / 355$ & \\
\hline \multicolumn{7}{|l|}{ Recurrence } \\
\hline No & $17 / 314$ & $215 / 314$ & \multirow[t]{2}{*}{1.000} & $96 / 356$ & $171 / 356$ & \multirow[t]{2}{*}{0.033} \\
\hline Yes & $6 / 314$ & $76 / 314$ & & $44 / 356$ & /356 & \\
\hline
\end{tabular}

${ }^{a}$ Manifestations including missing values represented in denominators.

\section{Discussion}

Significant correlations between MUC1 expression and clinicopathological manifestations including Lauren type or recurrence were not identified in the present study. In several studies, MUC1 expression has been suggested as a prognostic factor of gastric adenocarcinoma (1,22-24). The proportion of MUC1 overexpression in the present study was more than $90 \%$. However, MUC1 expression was detected in $30-60 \%$ of gastric adenocarcinoma cases in other studies. The difference in MUC1 expression could be explained by different conditions including the type of commercial antibody, concentration of the primary antibody or substrate of staining. Therefore, for higher accuracy, further studies should be undertaken with a larger number of gastric adenocarcinoma samples using standardized methods. Similarly, comparing the expression patterns of other molecules such as E-cadherin or other mucins may potentiate the possible use of MUC1 as a prognostic marker for gastric adenocarcinomas. However, we demonstrated that MUC1 expression may be valuable in the diagnosis of gastric adenocarcinoma particularly in difficult clinical cases including metastatic carcinoma of unknown primary site.

Aberrant expression of MUC4 has been reported in various cancers and inflammatory diseases. MUC4 is upregulated in high-grade dysplasia and adenocarcinoma of the esophagus. High-grade salivary tumors have a trend for reduced MUC4 expression compared to low-grade and intermediate-grade tumors. MUC4-expressing salivary gland mucoepidermoid tumors are associated with improved patient survival and a longer time to recurrence as compared with patients whose tumors were diagnosed as being negative for MUC4 expression. In addition, prostate cancer has exhibited downregulation of MUC4 expression in prostate carcinomas as compared with that in the normal/benign prostate regions. In contrast to the aforementioned studies, high MUC4 expression correlates with a short disease-free interval and a poor survival rate of small-sized lung adenocarcinomas, cholangiocarcinomas, epithelial ovarian carcinomas, pancreatic cancers and colorectal adenocarcinoma $(12-16,25,26)$. MUC4 overexpression in gastric cancer tissues compared with normal adjacent tissues has been previously reported (27). MUC4 is known to be expressed in embryonic gastric tissues at approximately 8 weeks gestation. It has also been shown that a number of embryogenesis phenomena such as cell proliferation, lineage allocation, cell migration, and differentiation of cells are also observed during cancer progression (28). Overexpression of MUC4 has a role in promoting properties in poorly differentiated gastric non-signet ring cell carcinoma cells (29). Our study into the role of MUC4 as a prognostic marker in gastric adenocarcinoma tissues found contradictory results. The level of MUC4 expression was higher in the intestinal-type than the diffuse-type but did not achieve statistical significance. Therefore, further research is required to evaluate the functional diversity of MUC4. MUC4 is an intramembrane ligand for receptor tyrosine kinase ErbB2, which is a transmembrane 
A

MUC4 expression

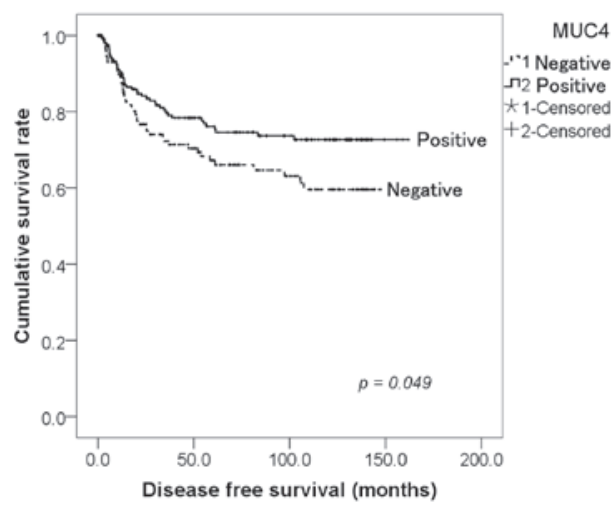

C

\section{MUC4 in intestinal type}

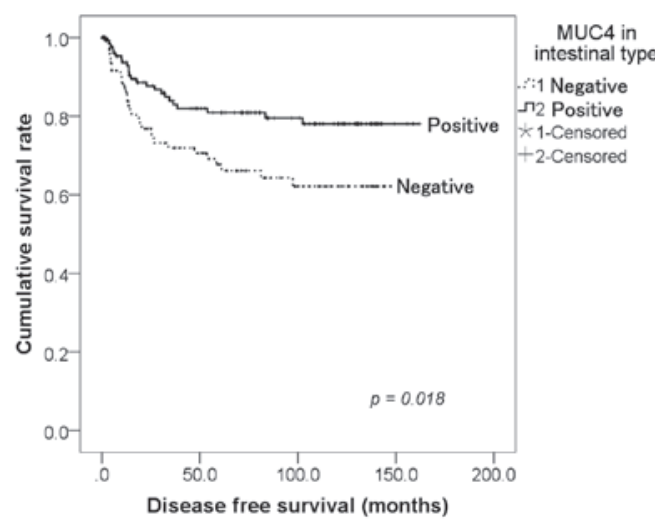

\section{MUC4 expression}

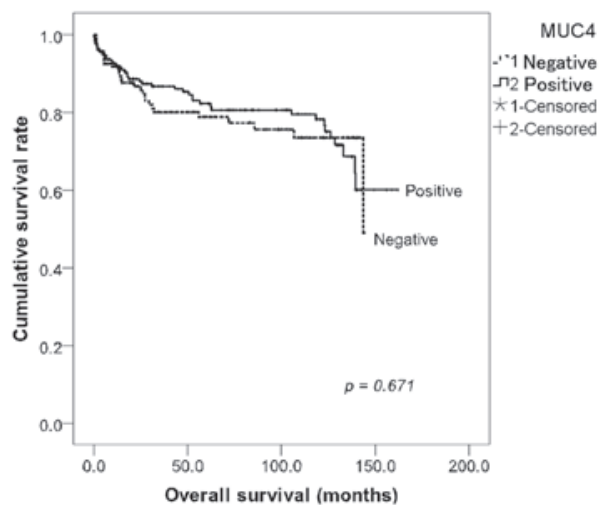

D

MUC4 in old age

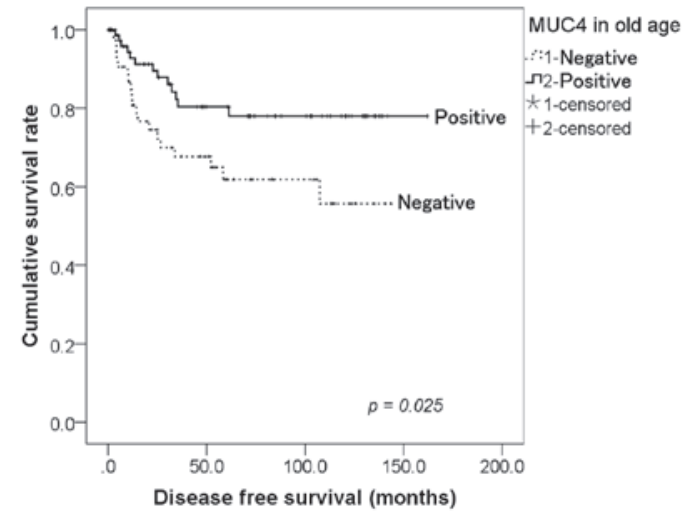

Figure 3. Disease-free and overall survival of patients with MUC4 expression and clinicopathological variables in gastric adenocarcinoma. (A) MUC4 expression was significantly correlated with better disease-free survival ( $\mathrm{p}=0.049$ ), while (B) MUC4 expression was not significantly correlated with overall survival $(\mathrm{p}=0.671)$. (C) MUC4 expression in intestinal-type or (D) in the older age group was significantly correlated with disease-free survival ( $\mathrm{p}=0.018$ and 0.025$)$.

glycoprotein encoded by the $c$-Erb-B2 proto-oncogene with a tyrosine kinase domain (13,30-32). MUC4 expression may be a more important marker in tumor differentiation than the cell signaling pathway or as an epithelial-mesenchymal transition relating factor. Likewise in gastric adenocarcinoma, MUC4 expression may be a more important tumor differentiation marker and loss of MUC4 expression may denote poor differentiation regardless of morphological change. Additionally, there are studies demonstrating that MUC4 expression was correlated with increased apoptosis. MUC4 was found to upregulate the expression of the cell cycle inhibitor p27 $7^{\mathrm{kip}}$. Hence, the role of MUC4 in apoptotic cell death is seemingly regulated by $E r b-B 2$ and other signaling components that are altered in the cancer cell (33).

Our study focused on investigating the prognostic significance of MUC1 and MUC4 expression in gastric cancer. We identified that loss of MUC4 expression was significantly correlated with worse disease-free survival. MUC4 may serve diverse functions in a context-dependent manner. There is a need to further our comprehension of the function and mechanism of MUC4 in normal and pathological conditions of gastric epithelial cells. Therefore, delineating the signaling mechanisms in the expression of MUC4 and defining the specific functions of MUC4 may lead to a better understanding of gastric adenocarcinoma.

\section{References}

1. Li XH, Zheng HC, Wang ZG, et al: The clinicopathological and prognostic significance of MUC-1 expression in Japanese gastric carcinomas: an immunohistochemical study of tissue microarrays. Anticancer Res 28: 1061-1067, 2008.

2. Hollingsworth MA and Swanson BJ: Mucins in cancer: protection and control of the cell surface. Nat Rev Cancer 4: 45-60, 2004.

3. Zheng H, Takahashi H, Nakajima T, et al: MUC6 down-regulation correlates with gastric carcinoma progression and a poor prognosis: an immunohistochemical study with tissue microarrays. J Cancer Res Clin Oncol 132: 817-823, 2006.

4. Cozzi PJ, Wang J, Delprado W, et al: MUC1, MUC2, MUC4, MUC5AC and MUC6 expression in the progression of prostate cancer. Clin Exp Metastasis 22: 565-573, 2005.

5. Fattorossi A, Battaglia A, Malinconico P, et al: Constitutive and inducible expression of the epithelial antigen MUC1 (CD227) in human T cells. Exp Cell Res 280: 107-118, 2002.

6. Pandey P, Kharbanda S and Kufe D: Association of the DF3/ MUC1 breast cancer antigen with Grb2 and the Sos/Ras exchange protein. Cancer Res 55: 4000-4003, 1995.

7. Monges GM, Mathoulin-Portier MP, Acres RB, et al: Differential MUC 1 expression in normal and neoplastic human pancreatic tissue. An immunohistochemical study of 60 samples. Am J Clin Pathol 112: 635-640, 1999.

8. Nakamori S, Ota DM, Cleary KR, Shirotani K and Irimura T: MUC1 mucin expression as a marker of progression and metastasis of human colorectal carcinoma. Gastroenterology 106: 353-361, 1994.

9. Khodarev NN, Pitroda SP, Beckett MA, et al: MUC1-induced transcriptional programs associated with tumorigenesis predict outcome in breast and lung cancer. Cancer Res 69: 2833-2837, 2009. 
10. Moniaux N, Escande F, Porchet N, Aubert JP and Batra SK: Structural organization and classification of the human mucin genes. Front Biosci 6: D1192-D1206, 2001.

11. Ho SB, Niehans GA, Lyftogt C, et al: Heterogeneity of mucin gene expression in normal and neoplastic tissues. Cancer Res 53: 641-651, 1993.

12. Jeon JM, Lee HW, Park JY, et al: Expression of MUC1 and MUC4 and its prognostic significance in non-small cell lung carcinoma. Korean J Pathol 44: 397-403, 2010.

13. Shibahara H, Tamada S, Higashi M, et al: MUC4 is a novel prognostic factor of intrahepatic cholangiocarcinoma-mass forming type. Hepatology 39: 220-229, 2004.

14. Tamada S, Shibahara H, Higashi M, et al: MUC4 is a novel prognostic factor of extrahepatic bile duct carcinoma. Clin Cancer Res 12: 4257-4264, 2006.

15. Saitou M, Goto M, Horinouchi M, et al: MUC4 expression is a novel prognostic factor in patients with invasive ductal carcinoma of the pancreas. J Clin Pathol 58: 845-852, 2005.

16. Tsutsumida H, Goto M, Kitajima S, et al: MUC4 expression correlates with poor prognosis in small-sized lung adenocarcinoma. Lung Cancer 55: 195-203, 2007.

17. Singh AP, Chauhan SC, Bafna S, et al: Aberrant expression of transmembrane mucins, MUC1 and MUC4, in human prostate carcinomas. Prostate 66: 421-429, 2006

18. Kwon KY, Ro JY, Singhal N, et al: MUC4 expression in non-small cell lung carcinomas: relationship to tumor histology and patient survival. Arch Pathol Lab Med 131: 593-598, 2007.

19. Weed DT, Gomez-Fernandez C, Pacheco J, et al: MUC4 and ERBB2 expression in major and minor salivary gland mucoepidermoid carcinoma. Head Neck 26: 353-364, 2004.

20. Alos L, Lujan B, Castillo M, et al: Expression of membranebound mucins (MUC1 and MUC4) and secreted mucins (MUC2, MUC5AC, MUC5B, MUC6 and MUC7) in mucoepidermoid carcinomas of salivary glands. Am J Surg Pathol 29: 806-813, 2005.

21. Weed DT, Gomez-Fernandez C, Yasin M, et al: MUC4 and ErbB2 expression in squamous cell carcinoma of the upper aerodigestive tract: correlation with clinical outcomes. Laryngoscope 114: $1-32,2004$

22. Yonezawa S, Goto M, Yamada N, Higashi M and Nomoto M: Expression profiles of MUC1, MUC2, and MUC4 mucins in human neoplasms and their relationship with biological behavior. Proteomics 8: 3329-3341, 2008.
23. Akyurek N, Akyol G, Dursun A, Yamac D and Gunel N: Expression of MUC1 and MUC2 mucins in gastric carcinomas: their relationship with clinicopathologic parameters and prognosis. Pathol Res Pract 198: 665-674, 2002.

24. Utsunomiya T, Yonezawa S, Sakamoto H, et al: Expression of MUC1 and MUC2 mucins in gastric carcinomas: its relationship with the prognosis of the patients. Clin Cancer Res 4: 2605-2614, 1998.

25. Westgaard A, Schjolberg AR, Cvancarova M, Eide TJ, Clausen OP and Gladhaug IP: Differentiation markers in pancreatic head adenocarcinomas: MUC1 and MUC4 expression indicates poor prognosis in pancreatobiliary differentiated tumours. Histopathology 54: 337-347, 2009.

26. Shanmugam C, Jhala NC, Katkoori VR, et al: Prognostic value of mucin 4 expression in colorectal adenocarcinomas. Cancer 116 : 3577-3586, 2010.

27. Lopez-Ferrer A, de Bolos C, Barranco C, et al: Role of fucosyltransferases in the association between apomucin and Lewis antigen expression in normal and malignant gastric epithelium. Gut 47: 349-356, 2000.

28. Buisine MP, Devisme L, Maunoury V, et al: Developmental mucin gene expression in the gastroduodenal tract and accessory digestive glands. I. Stomach. A relationship to gastric carcinoma. J Histochem Cytochem 48: 1657-1666, 2000.

29. Senapati S, Chaturvedi P, Sharma P, et al: Deregulation of MUC4 in gastric adenocarcinoma: potential pathobiological implication in poorly differentiated non-signet ring cell type gastric cancer. Br J Cancer 99: 949-956, 2008.

30. Moniaux N, Nollet S, Porchet N, Degand P, Laine A and Aubert JP: Complete sequence of the human mucin MUC4: a putative cell membrane-associated mucin. Biochem J 338: 325-333, 1999.

31. Carraway KL III, Rossi EA, Komatsu M, et al: An intramembrane modulator of the ErbB2 receptor tyrosine kinase that potentiates neuregulin signaling. J Biol Chem 274: 5263-5266, 1999.

32. Carraway KL, Ramsauer VP, Haq B and Carothers Carraway CA: Cell signaling through membrane mucins. Bioessays 25: 66-71, 2003.

33. Jepson S, Komatsu M, Haq B, et al: Muc4/sialomucin complex, the intramembrane ErbB2 ligand, induces specific phosphorylation of ErbB2 and enhances expression of p27(kip), but does not activate mitogen-activated kinase or protein kinaseB/Akt pathways. Oncogene 21: 7524-7532, 2002. 